\title{
Adopting Blockchain Technologies in Cloud for Efficient Data Storage and Enhanced Security
}

\author{
Veena, Ahmed Mudassar Ali, Ananthi, Gowri, Sureka
}

\begin{abstract}
In recent years, an innovative and potential technology called the blockchain has entered the developers community. The cloud storage has enabled the ground breaking development with several constraints associated with storage, security and cost. The block chain technology overcomes the cloud's most threatening distress security issues. The important factor is that cloud replaced the legacy enterprise solutions. Similarly, the decentralized ledgers will substitute the centralized cloud. This technology has solved the query raised by the majority of the enterprise executives by simply providing a better way to prevent forgery of the data with enhanced security.
\end{abstract}

Index Terms- Block chain, Crypto currency, Distributed system, Digital ledger, Ethereum.

\section{INTRODUCTION}

Over the period of time, data storage has become a significant issue. The radical improvement in the technology has led to the increasing demand for the digital resources. The concern for the cloud security over these enormous data is highly rising. Data stored in the cloud is highly prone to malicious attack that may result in disclosing of the data. Because of the meagreness in the client's trust the users are focussing on the advancement in the security of the cloud environment. In order to support the digital society and to surpass the security issues in cloud we are now using block chain technology.

The innovation level of this technology is highly promising that it will be into the mainstream sooner. The storage system ensures that the data stored is highly confidential which is protected under high security. There are still gruelling situations which need to overcome the high level of processing integrity.

The main aim of favouring this technology is to solve the integrity and security problem without the use of the central server. No single entity can have the control over the data since each and every single transaction is correlated. The identity of the participants in the block chain is not disclosed as there is no trusted entity to authenticate the participating users.

Therefore, the security relies on public verifiability of its integrity. It is a tamper-resistant database storage of the number of data nodes. This technology has the biggest effect

on trust. Permanent records are maintained by sequentially connecting the newer blocks of record with the older one.
This method of chaining the data in the blocks makes it a trust machine where the data cannot be erased or changed. Making the smart move towards the decentralized technology is definitely a development towards the future.

\section{SECURITY ISSUES IN TRADITIONAL SYSTEMS}

There are numerous advantages in cloud computing but on a recent report it has been surveyed that only $33 \%$ of the IT companies are completely dependent on the cloud environment. The rest of them have reported that they are facing several serious security issues once the data leaves their firewall.

In recent times, we have also been continuously cautioned about the data breaches over the social networks. There is an increasing sophisticated attacks which targets on stealing the corporate data which is found to be the major backdrop of this adoption. The software industries are very much specific of their client's data and the company's privacy. Inorder to prevent the data breach the companies need to implement the encryption techniques over their data before sending them to cloud environment. This makes most of the companies to be more hesitant to use cloud.

It is highly impossible to prevent the man made errors. but still recovering the stolen data from the default cloud pushes the client to a challenging situation. Poor identity and login management has to be resolved since the intruders can easily hack the password with minimal effort. This requires the usage of encrypted passwords. There is a possibility of Denial-of-Service attack that impacts the speed of the user's machine and prohibits the access to the applications and other data so that the user cannot access the information.

\section{BLOCK CHAIN TECHNOLOGIES}

The drawbacks of the cloud computing can be overcome by implementing the blockchain. This technology works where the data is stored in the blocks which is similar to writing on a notebook.Different data such as medical records, public polling, property agreements, etc., Here each block is tied to the previous block by embedding the current block with the previous block's hash value. Whenever an intruder tries to tamper the data which indirectly breaks the chain. Therefore the data security is high and the data is immutable. The system management has been designed in such a way
Revised Version Manuscript Received on August 19, 2019.

S.Veena, Professor, (email: veenas@saec.ac.in)

Ahmed Mudassar Ali, Professor, (email: ahmedmudassarali@saec.ac.in)

S.N.Ananthi, Assistant Professor, (email: ananthisn@saec.ac.in)

S.Veena, Assistant Professor, (email: gowrig@saec.ac.in)

V.Sureka, Assistant Professor, (email: sureka@saec.ac.in) 


\section{Adopting Blockchain Technologies in Cloud for Efficient Data Storage and Enhanced Security}

that it provides the capability to create, modify and monitor the components based on the user requirements. It also provides in-built integrity checking. Immutable shared view is provided so that no single entity can have control over the data. Dubai is the first to implement blockchain on a wide basis across their cities. A distributed ledger is maintained which maintains all the complete details of the transactions of the user.

Since it is the future of computing it takes a different approach for data processing and data storage with a different perspective from other technologies for security purposes. The traditional approach provides only the integrity and confidentiality but, blockchain provides availability and data integrity. DoS attack is difficult to implement in blockchain since it provides endpoint security. This can be deployed as permission less and permission that can be fairly termed as public and private blockchains respectively.

\section{COMPARISON OF EXISTING BLOCKCHAIN TECHNOLOGIES \& RESULTS}

a. Application of Asset Securitization and Block Chain of Internet Financial Firms

Tools : Block chain, Cloud computing

Advantages :If the duplicate file has the problem it can be restored normally.

Disadvantages : Needs improvement in the content and operation procedure of block payload

b. An automated system recovery using block chain technology

Tools : Block chain

Advantages :If the duplicate file has the problem it can be restored normally.

Disadvantages: Needs improvement in the content and operation procedure of block payload

\section{c. ChainFS : Block chain-secured cloud storage}

Tools : Block chain

Advantages : This system enables the end user to securely share the data in the cloud

Disadvantages : The latency need to be improved. The overhead increases as the file grows large

\section{d. Ethereum transaction graph analysis}

Tools : Ethereum, Block chain

Advantages : Crypto currency exchanges suggests thatit may be beneficial to link the ethereum transaction with bitcoin transaction. Provides additional insights to see if ETH was converted into BTC by hackers

Disadvantages: Requires deep understanding of the Ethereum platform for better results compared to graph analysis.

e. Resource transaction framework based on block chain in social community

Tools : Block chain, Smart contracts

Advantages : Ensures safe and secure transaction using smart contracts

Disadvantages: It is not scalable and cannot accommodate

\author{
large number of users
}

\section{f. Advanced block-chain architecture for e-health systems}

Tools : Block chain

Advantages : New cost-effective analysis and ease of access for the public. Medical researchers are open to access

Disadvantages: The cost of implementation is high. Migrating the existing records is difficult

g. Implementation of IoT system using block chain with authentication and data protection

Tools : Block chain, IoT

Advantages :Enhances security of data using Zero knowledge proof

Disadvantages: Prone to forgery and alteration of data and errors in the calculation of Charges

h. Block chain for large-scale internet of things data storage and protection

Tools : Blockchain, IoT

Advantages : Eliminates the use of a centralized server Disadvantages: For a system with complicated access control policies, more comprehensive designs need to be explored

i. Outsourcing service fair payment based on blockchain and its applications in cloud computing

$\begin{array}{ll}\text { Tools } & \text { : Block chain, Cloud computing } \\ \text { Advantages } & \text { BPay achieves soundness and robust } \\ & \text { fairness if the hash function is } \\ & \text { collision-resistant and ECDSA is } \\ \text { unforgeable. } & \text { BPay is efficient interms of the } \\ \text { computation cost } & \\ \text { Disadvantages: } & \text { The issue of payment fairness based o } \\ & \text { blockchain technologies in more } \\ & \text { complex cloud applications such as } \\ & \text { attribute-based data sharing is not } \\ & \text { addressed. }\end{array}$

j. A blockchain-based access control system for cloud storage

Tools : Blockchain, Cloud computing, Ethereum, Smart contracts

Advantages : Implements the access control model of the system to data stored in untrusted environments.

Disadvantages: Rejection of the fact and the inability to edit the data

k. Secured Data Storage Scheme based on Block Chain for Agricultural Products Tracking

Tools : Blockchain, double-chain storage, Internet of Things

Advantages : Double- chained server structure for security and query purpose. Solves the problem of storing custom data 
Disadvantages : Due to the transaction limit of the blockchain, there is a bottleneck in data throughput

\section{l. CloudPoS: A Proof-of-Stake Consensus Design for} Blockchain Integrated Cloud

Tools : Cryptocurrency, blockchain, PoS, Cloud computing

Advantages : The cloud environment is provided with the Blockchain-as-a-service

Disadvantages: The cloud service provider cannot be eliminated. The validators stake their resources for a fixed time which is required to overcome the bottleneck.

m.Saranyu: Using Smart Contracts and Blockchain for Cloud Tenant Management

Tools : Blockchain, smart contract and cryptocurrency

Advantages : This system is an excellent match with developing distributed cloud architectures. It has the ability to craft custom smart contracts for specific set of users

Disadvantages: It is not suitable for large scale cases. Need to be enhanced for more complex provisioning services

n. Block chain based data security enhanced IoT Server Platform

Tools

: Ethereum, blockchain, IoT server platform

Advantages : It provides enhanced security, makes use of the smart contract, encryption and authentication methods are used.

Disadvantages: Utility fee system service method requires enhanced security

o. Democratic Centralism: a hybrid Blockchain architecture and its applications in Energy Internet

Tools : Hybrid blockchain, security services, Energy internet

Advantages : This storage mechanism has shown improved results in high efficiency, intelligence and resource optimization

Disadvantages: Need to be improve the storage structure and constantly improve the transaction processing

\section{p. Beyond Bitcoin: The Rise of Blockchain World}

Tools : Ethereum, Blockchain, cryptocurrency

Advantages : Decentralization and autonomy are enabled by the blockchain and the choice can be made based on the needs of the application

Disadvantages: This technology doesn't guarantee that a blockchain cannot be corrupted

q. Blockchain Technology: Transforming Libertarian Cryptocurrency Dreams to Finance and Banking Realities

Tools : Bitcoin, blockchain, cryptocurrency

Advantages : Hybrid protocols overcomes the latency committee

barrier that replaces a single leader with a

Disadvantages: Bitcoin's security relies on servers wasting energy at an alarming rate and so there isa need to explore for an alternative

\section{CONCLUSION}

In this paper, the security and the data storage issues in the cloud computing is discussed along with the solution that can be implemented using the block chain technology. The paper also presented the need of the software industries to drive towards the block chain technology. Finally a comparison table is presented with all the existing methodologies that are tabulated and mentioned along with their advantages and drawbacks. The paper is concluded that the data security and storage space should be provided with minimum computational overhead which can be achieved by block chain technology.

\section{REFERENCES}

1. SuhwanBae and Yongtae Shin, An automated system recovery using Block chain, IEEE transaction 2018, ICUFN 2018.

2. Xiao Wan, Qingfan $\mathrm{Hu}$, Zheming Lu, Manlian $\mathrm{Yu}$, Application of asset securitization and Block chain of Internet financial firms, Nanjing University of Science and Technology, Nanjing, China.

3. QiwuZou, Yuzhe Tang, Ju Chen, Kai Li, ChainFS: Blockchain- secured cloud storage, IEEE cloud computing 2018.

4. KeGu, Linyu Wang, Bo Yin, Resource transaction framework based on block chain in social community, IEEE SmartWorld 2018, Ubiquitous intelligence \& computing, Internet of people and smart city innovations.

5. W. Liu, S.S. Zhu, T. Mundie, U.Kreiger, Advanced Block chain architecture for e-health systems, $201719^{\text {th }}$ International conference on E-health networking, Applications \& Services.

6. Chan Hyeok Lee, Ki Hyung, Kim, Implementation of IoT system using Block chain with authentication and data protection, IEEE 2018

7. Ruinian Li, Tianyi Song, Bo Mei, Hong Li, Block chain for large-scale internet of things data storage and protection, IEEE Transactions on Service Computing 2018.

8. Yinghui Zhang, Robert H. Deng, Ximeng Liu, Dong Zheng, Outsourcing service fair payment based on Block chain and its applications in cloud computing, IEEE transactions on services computing, 2018.

9. IlyaSukhodolskiy, Sergey Zapechnikov, A block chain based access control system for cloud storage, IEEE Transaction 2018.

10. Chao Xie, Yan Sun, Hong Luo, Secured data storage scheme based on Block chain for agricultural products tracking, $20173^{\text {rd }}$ International Conference on Big data computing and communications. 